Comment. Math. Helv. 75 (2000) 668-680

(C) 2000 Birkhäuser Verlag, Basel

$0010-2571 / 00 / 040668-13 \$ 1.50+0.20 / 0$

Commentarii Mathematici Helvetici

\title{
Weierstrass representation of Lagrangian surfaces in four-dimensional space using spinors and quaternions
}

\author{
Frédéric Hélein and Pascal Romon
}

\begin{abstract}
We derive a Weierstrass-type formula for conformal Lagrangian immersions in Euclidean 4-space, and show that the data satisfies an equation similar to Dirac equation with complex potential. Alternatively this representation has a simple formulation using quaternions. We apply it to the Hamiltonian stationary case and construct all possible tori, thus obtaining a first approach to a moduli space in terms of a simple algebraic-geometric problem on the plane. We also classify Hamiltonian stationary Klein bottles and show they self-intersect.
\end{abstract}

Mathematics Subject Classification (2000). Primary 53C42, 53D12, Secondary 49Q10, $53 \mathrm{~A} 05$.

Keywords. Lagrangian surfaces, Weierstrass representation, Dirac equation, minimal surfaces, variational problem with constraint.

\section{Introduction}

In this paper we revisit and generalize some aspects of the Weierstrass construction for Hamiltonian stationary Lagrangian surfaces which was given in [HR]. We are interested here in Lagrangian surfaces in $\mathbb{R}^{4} \simeq \mathbb{C}^{2}$, equipped with the standard symplectic form, a priori without the assumption of being Hamiltonian stationary. In order to build a Weierstrass representation of these immersions, it is natural in a first step to consider the set of weakly conformal Lagrangian immersions of a Riemann surface: they are maps which coincide with a conformal immersion outside isolated points where the Jacobian matrix vanishes, and such that the pull-back of the symplectic form vanishes. In [HR], we analyzed weakly conformal Lagrangian immersions which are Hamiltonian stationary, i.e. critical points of the area functional with the requirements of (i) being Lagrangian and (ii) the only infinitesimal variations allowed are the one given by Hamiltonian vector fields (see $[\mathrm{O} 1],[\mathrm{O} 2],[\mathrm{SW}]$ ). They enjoy a nice characterization using the canonical Lagrangian angle map defined over any Lagrangian surface and with values in the circle $S^{1}$ (it is actually a part of the classical Gauss map of the immersion). A precise definition is given in Section 1 below. A Lagrangian surface is Hamiltonian 
stationary if and only if the Lagrangian angle map is a harmonic map from the surface to the circle. This leads to many nice properties which may be summarized by saying that these surfaces are solutions of a completely integrable system.

In the first Section, we show that the Hamiltonian stationary assumption may be removed and that we still have a Weierstrass type representation of all weakly conformal Lagrangian immersions. This is very similar to the Weierstrass representation for weakly conformal immersions in $\mathbb{R}^{3}$ due to B. G. Konopelchenko [Ko]. Some variants were proposed also in $[\mathrm{Ke}]$ and this representation has been studied by many authors [KoT1, KoT2], [B1, B2], [KuS], [T1, T2]. Actually we shall first present our representation using notations that make evident the similarities between the two theories, since they rely on a kind of Dirac equation.

In the second Section, we propose an equivalent representation using quaternions. The gain is not only formal, but it also unveils the quaternionic structure of the problem. In particular when we specialize back to Hamiltonian stationary Lagrangian immersions, we see immediately that the set of solutions has the structure of a vector space over the quaternions. Exploiting this structure leads to formulae equivalent to the one in $[\mathrm{HR}]$ but much simpler to handle.

In the last Section, we present computations using these formulae and we focus on immersed Klein bottles. We classify all such surfaces and show that none is embedded.

\section{Acknowledgment}

The Authors are pleased to thank Claude Viterbo for interesting discussions.

\section{Weierstrass representation for Lagrangian surfaces in $\mathbb{C}^{2}$}

Let $\left(\epsilon_{1}, \epsilon_{2}\right)$ be the canonical basis of $\mathbb{C}^{2}$ over $\mathbb{C}$. We equipp $\mathbb{C}^{2}$ with the Hermitian product

$$
\langle v, w\rangle_{H}=v^{1} \overline{w^{1}}+v^{2} \overline{w^{2}}
$$

so that, as a real four-dimensional space, $\mathbb{C}^{2}$ has the Euclidean scalar product $\langle., .\rangle_{E}$ and the symplectic form $\omega$ given by

$$
\langle v, w\rangle_{H}=\langle v, w\rangle_{E}-i \omega(v, w)
$$

Let $\Omega \subset \mathbb{C}$ be a simply connected domain and $X: \Omega \longrightarrow \mathbb{C}^{2}$ some conformal Lagrangian immersion. Letting $e^{f}:=\left|\frac{\partial X}{\partial x}\right|^{2}=\left|\frac{\partial X}{\partial y}\right|^{2}$, and $e_{1}:=e^{-f / 2} \frac{\partial X}{\partial x}, e_{2}:=$ $e^{-f / 2} \frac{\partial X}{\partial y}$, we have

$$
d X=e^{f / 2}\left(e_{1} d x+e_{2} d y\right)
$$


and $\left(e_{1}, e_{2}\right)$ is necessarily the orthonormal basis of some Lagrangian plane in $\mathbb{C}^{2}$. By (1) this condition amounts to $\left\langle e_{1}, e_{2}\right\rangle_{H}=0$. Hence, for all $(x, y) \in \Omega$, the components of $e_{1}(x, y)$ and $e_{2}(x, y)$ in the basis $\left(\epsilon_{1}, \epsilon_{2}\right)$ form a matrix

$$
e(x, y)=\left(\begin{array}{ll}
e_{1}^{1} & e_{2}^{1} \\
e_{1}^{2} & e_{2}^{2}
\end{array}\right)(x, y)
$$

which belongs to $U(2)$. Decompose $e(x, y)$ as

$$
e(x, y)=e^{i \beta / 2} g
$$

where $\beta \in \mathbb{R} / 2 \pi \mathbb{Z}$ and $g \in S U(2)$. Notice that such a decomposition is not unique; the other possible one is $e(x, y)=e^{i(\beta+2 \pi) / 2}(-g)$. But since $\Omega$ is simply connected, we can construct smooth functions $\beta: \Omega \longrightarrow \mathbb{R} / 2 \pi \mathbb{Z}$ and $g: \Omega \longrightarrow S U(2)$, such that (4) holds everywhere. The angle $\beta$ is called the Lagrangian angle function, is characterized by $d z^{1} \wedge d z^{2}\left(e_{1}, e_{2}\right)=e^{i \beta}$.

Let us focus on $(x, y) \longmapsto e^{f(x, y) / 2} g(x, y)$, a map with values in $\mathbb{R}_{+}^{*} \cdot S U(2)$. We remark that $\mathbb{R}_{+} . S U(2)$ is isomorphic to the set of quaternions $\mathbb{H}$. Namely, letting

$$
\mathbf{1}:=\left(\begin{array}{cc}
1 & 0 \\
0 & 1
\end{array}\right), I:=\left(\begin{array}{cc}
0 & -1 \\
1 & 0
\end{array}\right), J:=\left(\begin{array}{cc}
i & 0 \\
0 & -i
\end{array}\right), K:=\left(\begin{array}{cc}
0 & i \\
i & 0
\end{array}\right)
$$

the map

$$
\begin{aligned}
\mathcal{H}: \quad \mathbb{R}_{+} \cdot S U(2) & \longmapsto \mathbb{H} \\
t 1+x I+y J+z K & \longmapsto t+x i+y j+z k
\end{aligned}
$$

is a field isomorphism ${ }^{1}$. Using the fact that each quaternion $\zeta$ can be written in an unique way $\zeta=a+j b$, where $a, b \in \mathbb{C} \subset \mathbb{H}$, we shall define two functions $s_{1}, s_{2}: \Omega \longrightarrow \mathbb{C}$ such that

$$
\mathcal{H}\left(e^{f(x, y) / 2} g(x, y)\right)=s_{1}(x, y)+j s_{2}(x, y)=s_{1}(x, y)+\overline{s_{2}}(x, y) j
$$

A computation shows that

$$
e^{f(x, y) / 2} g(x, y)=\left(\begin{array}{cc}
\operatorname{Re}\left(s_{1}\right)+i \operatorname{Re}\left(s_{2}\right) & -\operatorname{Im}\left(s_{1}\right)-i \operatorname{Im}\left(s_{2}\right) \\
\operatorname{Im}\left(s_{1}\right)-i \operatorname{Im}\left(s_{2}\right) & \operatorname{Re}\left(s_{1}\right)-i \operatorname{Re}\left(s_{2}\right)
\end{array}\right)(x, y) .
$$

1 One could also use the more natural isomorphism $\tilde{\mathcal{H}}:\left(\begin{array}{cc}a & -\bar{b} \\ b & \bar{a}\end{array}\right) \longmapsto a+j b$. Both isomorphisms are actually conjugate through $\mathcal{H}()=.\tau \tilde{\mathcal{H}} \tau^{-1}$, where $\tau:=\frac{\sqrt{2}}{2}(i+j)$. But $\mathcal{H}$ will be more suitable in the following. 
We deduce from (2) and (4) that in the basis $\left(\epsilon_{1}, \epsilon_{2}\right)$,

$$
\begin{aligned}
d X=e^{i \beta / 2}( & \left(\begin{array}{c}
\operatorname{Re}\left(s_{1}\right)+i \operatorname{Re}\left(s_{2}\right) \\
\operatorname{Im}\left(s_{1}\right)-i \operatorname{Im}\left(s_{2}\right)
\end{array}\right) d x \\
& \left.+\left(\begin{array}{c}
-\operatorname{Im}\left(s_{1}\right)-i \operatorname{Im}\left(s_{2}\right) \\
\operatorname{Re}\left(s_{1}\right)-i \operatorname{Re}\left(s_{2}\right)
\end{array}\right) d y\right) \\
= & e^{i \beta / 2}\left(\operatorname{Re}\left[\left(\begin{array}{c}
s_{1} \\
-i s_{1}
\end{array}\right) d z\right]+i \operatorname{Re}\left[\left(\begin{array}{c}
s_{2} \\
i s_{2}
\end{array}\right) d z\right]\right) .
\end{aligned}
$$

We look for the necessary and sufficient conditions on $\left(s_{1}, s_{2}\right)$ such that a solution of (5) exists on all simply-connected domain, i. e.

$$
\begin{aligned}
0 & =d\left(e^{i \beta / 2} \operatorname{Re}\left[\left(\begin{array}{c}
s_{1} \\
-i s_{1}
\end{array}\right) d z\right]+i e^{i \beta / 2} \operatorname{Re}\left[\left(\begin{array}{c}
s_{2} \\
i s_{2}
\end{array}\right) d z\right]\right) \\
& =e^{i \beta / 2}\left(\begin{array}{c}
-\operatorname{Im}\left(\frac{\partial \overline{s_{2}}}{\partial z}-\frac{1}{2} \frac{\partial \beta}{\partial \bar{z}} s_{1}\right)-i \operatorname{Im}\left(\frac{\partial s_{1}}{\partial \bar{z}}+\frac{1}{2} \frac{\partial \beta}{\partial z} \overline{s_{2}}\right) \\
\operatorname{Re}\left(\frac{\partial \overline{s_{2}}}{\partial z}-\frac{1}{2} \frac{\partial \beta}{\partial \bar{z}} s_{1}\right)+i \operatorname{Re}\left(\frac{\partial s_{1}}{\partial \bar{z}}+\frac{1}{2} \frac{\partial \beta}{\partial z} \overline{s_{2}}\right)
\end{array}\right) d z \wedge d \bar{z}
\end{aligned}
$$

We see that (6) is true if and only if

$$
\left\{\begin{array}{l}
\frac{\partial s_{1}}{\partial \bar{z}}=-\frac{1}{2} \frac{\partial \beta}{\partial z} \overline{s_{2}} \\
\frac{\partial \overline{s_{2}}}{\partial z}=\frac{1}{2} \frac{\partial \beta}{\partial \bar{z}} s_{1} .
\end{array}\right.
$$

This system may be written using a kind of Dirac operator. Set

$$
U:=\frac{1}{2} \frac{\partial \beta}{\partial z}, \quad \phi:=\left(\begin{array}{c}
s_{1} \\
s_{2}
\end{array}\right), \quad \mathcal{D}:=\left(\begin{array}{cc}
0 & \frac{\partial}{\partial z} \\
-\frac{\partial}{\partial \bar{z}} & 0
\end{array}\right),
$$

then (7) is equivalent to the following

$$
\mathcal{D} \phi=\left(\begin{array}{cc}
\bar{U} & 0 \\
0 & U
\end{array}\right) \phi
$$

Conversely one may check directly that, for any smooth function $\beta: \Omega \longrightarrow \mathbb{R} / 2 \pi \mathbb{Z}$, any solution $\phi=\left(\frac{s_{1}}{s_{2}}\right)$ of $(8)$ produces a $\mathbb{C}^{2}$-valued closed 1 -form on $\Omega$ given by

$$
\alpha:=e^{i \beta / 2}\left(\operatorname{Re}\left[\left(\begin{array}{c}
s_{1} \\
-i s_{1}
\end{array}\right) d z\right]+i \operatorname{Re}\left[\left(\begin{array}{c}
s_{2} \\
i s_{2}
\end{array}\right) d z\right]\right) .
$$

And if $\phi \neq 0$, any solution $X: \Omega \longrightarrow \mathbb{C}^{2}$ of $d X=\alpha$ is a conformal Lagrangian immersion, with Lagrangian angle map $\beta$. Thus we obtain the following 
Theorem 1. Let $\Omega \subset \mathbb{C}$ be a simply connected domain. For any conformal Lagrangian immersion $X: \Omega \longrightarrow \mathbb{C}^{2}$, there exist smooth functions $\beta: \Omega \longrightarrow$ $\mathbb{R} / 2 \pi \mathbb{Z}$ and $\phi=\left(\frac{s_{1}}{s_{2}}\right): \Omega \longrightarrow \mathbb{C}^{2} \backslash\{0\}$, determined by (5) and then $\phi$ is a solution of the Dirac equation (8). Conversely any never vanishing solution of (8) gives rise to a conformal Lagrangian immersion obtained by integrating (5).

Remark that the case where $\phi$ vanishes on isolated points corresponds to weakly conformal immersions. All this construction can actually be understood in terms of a decomposition of the complexification of the Hermitian Galilee group of $\mathbb{C}^{2}$, $U(2) \ltimes \mathbb{C}^{2}$ by an order fourth automorphism (see [HR] for details).

It is tempting to compare this result with the spinorial Weierstrass representation due to B. G. Konopelchenko [Ko] of surfaces in $\mathbb{R}^{3}$ (see also [KoT1, $\mathrm{KoT} 2],[\mathrm{B} 1, \mathrm{~B} 2],[\mathrm{KuS}],[\mathrm{T} 1, \mathrm{~T} 2])$. Let $p: \Omega \longrightarrow \mathbb{R}$ be a smooth function and $\phi=\left(\frac{s_{1}}{s_{2}}\right): \Omega \longrightarrow \mathbb{C}^{2} \backslash\{0\}$ be a solution of the Dirac equation

$$
\mathcal{D} \phi=\left(\begin{array}{cc}
p & 0 \\
0 & p
\end{array}\right) \phi
$$

Then the following 1-form

$$
\eta:=\operatorname{Re}\left(\begin{array}{c}
s_{1}^{2} d z-{\overline{s_{2}}}^{2} d \bar{z} \\
-i\left(s_{1}^{2} d z-{\overline{s_{2}}}^{2} d \bar{z}\right) \\
s_{1} s_{2} d z+\bar{s}_{1} s_{2} d \bar{z}
\end{array}\right)
$$

is closed and any solution $Y: \Omega \longrightarrow \mathbb{R}^{3}$ of the equation $d Y=\eta$ is a conformal immersion. Moreover the mean curvature of this surface satisfies

$$
2 p=H\left(\left|s_{1}\right|^{2}+\left|s_{2}\right|^{2}\right)
$$

and the pull-back metric is $\left(\left|s_{1}\right|^{2}+\left|s_{2}\right|^{2}\right)^{2} d z d \bar{z}$.

The similarity between the two algorithms is striking but not total, since the potentials $\left(\begin{array}{cc}p & 0 \\ 0 & p\end{array}\right)$ and $\left(\begin{array}{cc}\bar{U} & 0 \\ 0 & U\end{array}\right)$ generally differ. For an arbitrary function $U$ : $\Omega \longrightarrow \mathbb{C}$ and for a solution $\phi$ of

$$
\mathcal{D} \phi=\left(\begin{array}{cc}
\bar{U} & 0 \\
0 & U
\end{array}\right) \phi
$$

the relation (5), which gives rise to a map into $\mathbb{C}^{2}$, makes sense only if $U$ has the form $U=\frac{1}{2} \frac{\partial \beta}{\partial z}$, with $\beta$ real valued. And the Konopelchenko ansatz integrates only if $U$ is real. Thus there is an intersection case, where $U$ is both real valued and 
of the form $U=\frac{1}{2} \frac{\partial \beta}{\partial z}$, which is realized if and only if $\beta$ is a real valued function of the variable $x$. An interesting subcase is when $\beta(x, y)=4 x \Leftrightarrow U=p=1$. The corresponding surfaces in $\mathbb{R}^{3}$ have been studied by J. Richter $[R]$ in his thesis where there are named Dirac surfaces. Another intrinsic difference between the two representations becomes obvious at the global level: in Konopelchenko's case the quantities $s_{1}, s_{2}$ are spinors and the potential $p$ is a density, while in the Lagrangian case they are all 1-forms (though with a similar sign twist).

\section{Formulation using quaternions}

We identify $\mathbb{C}^{2}$ with the set of quaternions $\mathbb{H}$ using the real vector space isomorphism

$$
\begin{aligned}
& \Phi: \quad \mathbb{C}^{2} \longrightarrow \mathbb{H} \\
& z=\left(\begin{array}{c}
z^{1} \\
z^{2}
\end{array}\right) \longmapsto \tau\left(z^{1}+j z^{2}\right) \tau^{-1},
\end{aligned}
$$

where $\tau:=\frac{\sqrt{2}}{2}(i+j)$ and $\mathbb{C}=\mathbb{R}+i \mathbb{R} \subset \mathbb{R}+i \mathbb{R}+j \mathbb{R}+k \mathbb{R}=\mathbb{H}$. Notice that $\Phi\left(\epsilon_{1}\right)=1$ and $\Phi\left(\epsilon_{2}\right)=i$. Similarly we recall the field isomorphism already used in the previous section between $\mathbb{R}_{+} . S U(2)$ and $\mathbb{H}$,

$$
\begin{aligned}
\mathcal{H}: & \mathbb{R}_{+} \cdot S U(2) \longrightarrow \mathbb{H} \\
& \left(\begin{array}{cc}
a & -\bar{b} \\
b & \bar{a}
\end{array}\right) \\
& \longmapsto \tau(a+j b) \tau^{-1} .
\end{aligned}
$$

Then a short computation shows that $\forall e^{i \theta} \in S^{1} \subset \mathbb{C}, \forall R \in \mathbb{R}_{+} . S U(2)$ and $\forall z \in \mathbb{C}^{2}$,

$$
\Phi\left(e^{i \theta} R z\right)=\mathcal{H}(R) \Phi(z) e^{j \theta} .
$$

Thus, if $X: \Omega \longrightarrow \mathbb{C}^{2}$ and we denote $d X=e^{(f+i \beta) / 2}\left(g\left(\epsilon_{1}\right) d x+g\left(\epsilon_{2}\right) d y\right)$,

$$
d(\Phi \circ X)=\mathcal{H}\left(e^{f / 2} g\right)\left(\Phi\left(\epsilon_{1}\right) e^{j \beta / 2} d x+\Phi\left(\epsilon_{2}\right) e^{j \beta / 2} d y\right)=\mathcal{H}\left(e^{f / 2} g\right) d z e^{j \beta / 2},
$$

where $d z:=d x+i d y$ is viewed as $\mathbb{H}$-valued ${ }^{2}$. In the remainder of this section, we shall abuse notations and denote $X \simeq \Phi \circ X(X$ is then a map into $\mathbb{H})$ and we also set $h:=\mathcal{H}\left(e^{f / 2} g\right)$. Moreover in our computations, we shall assume that $\Omega \subset \mathbb{C} \subset \mathbb{H}$. Thus we define the Cauchy-Riemann operators $\partial z \backslash \partial, \partial \bar{z} \backslash \partial, \partial / \partial z$ and $\partial / \partial \bar{z}$, such that

$$
d h=(\partial h / \partial z) d z+(\partial h / \partial \bar{z}) d \bar{z}=d z(\partial z \backslash \partial h)+d \bar{z}(\partial \bar{z} \backslash \partial h) .
$$

\footnotetext{
2 The quaternionic notation may mislead the Reader into viewing $d(\Phi \circ X)$ as a $(1,0)$-form, since no $d \bar{z}$ term is present. That notion however is not valid, and we might introduce $d \bar{z}$ using the property $d z j=j d \bar{z}$.
} 
Hence the compatibility condition for a solution $X$ of $d X=h d z e^{j \beta / 2}$ to exist is

$$
\begin{aligned}
0 & =((\partial h / \partial z) d z+(\partial h / \partial \bar{z}) d \bar{z}) \wedge d z e^{j \beta / 2}-h d z \wedge d \beta \frac{j}{2} e^{j \beta / 2} \\
& =\left((\partial h / \partial \bar{z})-h \frac{\partial \beta}{\partial \bar{z}} \frac{j}{2}\right) d \bar{z} \wedge d z e^{j \beta / 2} .
\end{aligned}
$$

(We shall constantly use the fact that if $u \in \mathbb{C} \subset \mathbb{H}, j u=\bar{u} j$ and $k u=\bar{u} k$. Here this is reponsable for the signs in the formula, since $d \bar{z} \wedge d z=2 i d x \wedge d y$.) Hence the compatibility condition writes

$$
(\partial h / \partial \bar{z})=h \frac{\partial \beta}{\partial \bar{z}} \frac{j}{2} .
$$

If we let $h=s_{1}+j s_{2}$ and substitute in this equation, we see that $s_{1}, s_{2}$ are solutions of the system (7) or (8).

\subsection{Hamiltonian stationary Lagrangian immersions}

As proven in $[\mathrm{HR}]$, Hamiltonian stationary Lagrangian immersions are characterized in this framework by the additional condition that $\beta$ is a harmonic function. Thus they may be constructed by first picking up some harmonic function $\beta$ and second solving Equation (13), where $h: \Omega \longrightarrow \mathbb{H}$ is the unknown function. It is then clear that the set of solutions of this equation is a quaternionic vector space $(\mathbb{H}$ acting on the left).

Let us focus (as in $[\mathrm{HR}]$ ) on the toric solutions. Since any torus is conformally equivalent to some $\mathbb{C} / \Gamma$, where $\Gamma:=v_{1} \mathbb{Z}+v_{2} \mathbb{Z}$ is a lattice in $\mathbb{C}$, it amounts to looking for $\Gamma$-periodic Hamiltonian stationary Lagrangian immersions $X: \mathbb{C} \longrightarrow$ $\mathbb{H}$. Setting

$$
d X=h d z e^{j \beta / 2}
$$

we are led to look for (a priori) $2 \Gamma$-periodic maps $h: \mathbb{C} \longrightarrow \mathbb{H}$ and $\Gamma$-periodic maps $\beta: \mathbb{C} \longrightarrow \mathbb{R} / 2 \pi \mathbb{Z}$, such that $h e^{j \beta / 2}$ is $\Gamma$-periodic, $\beta$ is harmonic and $h$ is a solution of (13). The only such $\beta$ 's are of the form $\beta(z)=2 \pi\left\langle\beta_{0}, z-z_{0}\right\rangle=$ $\pi\left(\overline{\beta_{0}}\left(z-z_{0}\right)+\beta_{0}\left(\bar{z}-\overline{z_{0}}\right)\right)$, with $\beta_{0} \in \Gamma^{\star}$, where $\Gamma^{\star}$ is the dual lattice to $\Gamma$. Without loss of generality we shall assume that $z_{0}=0$. The $2 \Gamma$-periodic maps $h: \mathbb{C} \longrightarrow \mathbb{H}$ are of the form

$$
h=\sum_{\gamma \in \frac{1}{2} \Gamma^{\star}} \hat{h}_{\gamma} e^{i 2 \pi\langle\gamma, z\rangle},
$$

where $\hat{h}_{\gamma}$ are unique Fourier coefficients ${ }^{3}$ in $\mathbb{H}$. Now the equation (13) is written

\footnotetext{
3 Notice that we do not need here reality conditions on the Fourier coefficients $\hat{h}_{\gamma}$. A way to convince oneself that any $2 \Gamma$-periodic function $f: \mathbb{C} \longrightarrow \mathbb{H}$ has such a unique Fourier decomposition is to set $f=f_{1}+j f_{2}$, where $f_{1}$ and $f_{2}$ are complex valued and to Fourier decompose $f_{1}$ and $f_{2}$.
} 
as

$$
\sum_{\gamma \in \frac{1}{2} \Gamma^{\star}} \hat{h}_{\gamma} i \gamma e^{i 2 \pi\langle\gamma, z\rangle}=\sum_{\gamma \in \frac{1}{2} \Gamma^{\star}} \hat{h}_{-\gamma} \frac{j \overline{\beta_{0}}}{2} e^{i 2 \pi\langle\gamma, z\rangle},
$$

which is satisfied if and only if

$$
\hat{h}_{\gamma}=\hat{h}_{-\gamma} \frac{\beta_{0 \gamma}}{2|\gamma|^{2}} k, \forall \gamma \in \frac{1}{2} \Gamma^{\star} .
$$

The relation (14) implies $\hat{h}_{\gamma}=\hat{h}_{\gamma} \frac{\left|\beta_{0}\right|^{2}}{4|\gamma|^{2}}$. Hence all $\hat{h}_{\gamma}$ 's vanish except when $|\gamma|=$ $\frac{\left|\beta_{0}\right|}{2}$, and then $\frac{\beta_{0} \gamma}{2|\gamma|^{2}} k=\frac{2 \gamma}{\beta_{0}} k$. Conversely, as shown in [HR], the map $h$ yields after integration a $\Gamma$-periodic immersion if and only if the $\gamma^{\prime}$ s for which $\hat{h}_{\gamma}$ does not vanish belong to

$$
\Gamma_{\beta_{0}}^{\star}:=\left\{\gamma \in \frac{\beta_{0}}{2}+\Gamma^{\star} ;|\gamma|=\frac{\left|\beta_{0}\right|}{2}, \gamma^{2} \neq \frac{\beta_{0}^{2}}{4}\right\},
$$

and the relation (14) is true. We can describe the solutions by considering a subset containing half of the relevant frequencies $\gamma$

$$
\Gamma_{\beta_{0},+}^{\star}:=\left\{\gamma \in \Gamma_{\beta_{0}}^{\star} / \operatorname{Im}\left(\gamma \beta_{0}^{-1}\right)>0\right\},
$$

and then, thanks to (14),

$$
\begin{aligned}
d X & =\sum_{\gamma \in \Gamma_{\beta_{0},+}^{\star}} \hat{h}_{\gamma}\left(e^{i 2 \pi\langle\gamma, z\rangle}-\frac{2 \gamma}{\overline{\beta_{0}}} k e^{-i 2 \pi\langle\gamma, z\rangle}\right) d z e^{j \pi\left\langle\beta_{0}, z\right\rangle} \\
& =\sum_{\gamma \in \Gamma_{\beta_{0},+}^{\star}} \hat{h}_{\gamma} e^{i 2 \pi\langle\gamma, z\rangle}\left(1-\frac{2 \gamma}{\overline{\beta_{0}}} k\right) d z e^{j \pi\left\langle\beta_{0}, z\right\rangle}
\end{aligned}
$$

We can integrate explicitly this equation and we obtain

$$
X(z)=X_{0}+\sum_{\gamma \in \Gamma_{\beta_{0},+}^{\star}} \hat{h}_{\gamma} X_{\gamma}(z)
$$

where $X_{0}, \hat{h}_{\gamma} \in \mathbb{H}$ are constants and

$$
X_{\gamma}(z)=e^{i 2 \pi\langle\gamma, z\rangle} \frac{\beta_{0}+2 \gamma k}{2 \pi \operatorname{Im}\left(\gamma \bar{\beta}_{0}\right)} e^{j \pi\left\langle\beta_{0}, z\right\rangle} .
$$

Hence, given $\beta_{0}$, the set of doubly periodic Hamiltonian stationary Lagrangian weakly conformal maps having a Lagrangian angle function $2 \pi\left\langle\beta_{0}, z\right\rangle$ is a quaternionic vector space of dimension $1+\operatorname{Card}\left(\Gamma_{\beta_{0},+}^{\star}\right)$ over $\mathbb{H}$. 


\section{Construction of immersed Hamiltonian stationary Lagrangian Klein bottles}

We depict an abstract Klein bottle $\mathcal{K}$ as the complex plane $\mathbb{C}$ quotiented by the following (renormalized) group action: $z \mapsto z+i \tau$ and $z \mapsto \bar{z}+1$ where $\tau$ is a real number. Iterating the second relation, we get $z \mapsto z+2$, hence the torus $\mathbf{T}=\mathbb{C} / \Gamma$, where $\Gamma=2 \mathbb{Z} \oplus i \tau \mathbb{Z}$, is a rectangular orientable double cover of $\mathcal{K}$. We can now use the formalism in the previous section and characterize the immersed Klein bottles as giving rise to particular cases of immersed tori.

Theorem 2. There exist Lagrangian Hamiltonian stationary Klein bottles; they are never embedded.

The proof proceeds in three steps.

Step 1: The Lagrangian angle.

The dual lattice $\Gamma^{\star}$ is $\frac{1}{2} \mathbb{Z} \oplus \frac{i}{\tau} \mathbb{Z}$, and $\beta_{0}$ belongs to $\Gamma^{\star}$ so $\beta_{0}=\frac{m}{2}+i \frac{n}{\tau}$ for some integers $m, n$. Since $z \mapsto \bar{z}+1$ reverses orientation of the tangent plane, we need $\beta(\bar{z}+1) \equiv \beta(z)+\pi \bmod 2 \pi$.

$$
\begin{aligned}
\beta(\bar{z}+1) & =2 \pi\left\langle\beta_{0}, \bar{z}+1\right\rangle=\pi\left(m(x+1)-\frac{2 n}{\tau} y\right) \\
& =\beta(z)+m \pi-\frac{4 n \pi y}{\tau}
\end{aligned}
$$

which imposes $n=0$ and $m$ odd. Finally $\beta(z)=m \pi x$.

Step 2: The basis vectors.

Take $\gamma=\frac{a \tau+i 4 b}{4 \tau} \in \Gamma_{\beta_{0},+}^{\star}$ (recall that $\Gamma_{\beta_{0},+}^{\star} \subset \frac{\beta_{0}}{2}+\Gamma^{\star}$ so $a$ is odd, $b$ is a positive integer) and $|\gamma|=\left|\frac{\beta_{0}}{2}\right|$ reads

$$
\left(a^{2}-m^{2}\right) \tau^{2}+16 b^{2}=0
$$

We associate two linked frequencies $\gamma$ and $\gamma^{\prime}=\bar{\gamma}$ to write $\hat{h}_{\gamma} X_{\gamma}+\hat{h}_{\bar{\gamma}} X_{\bar{\gamma}}=: \hat{h}_{\gamma} Y_{\gamma}$, where

$$
\begin{aligned}
Y_{\gamma}(z)= & X_{\gamma}(z)+i^{m+a} \frac{a \tau+4 b i}{m \tau} X_{\gamma^{\prime}} \\
= & \frac{1}{2 \pi b m}\left(m \tau e^{\frac{i \pi}{2}\left(a x+\frac{4 b y}{\tau}\right)}+(a \tau+4 b i) k e^{\frac{-i \pi}{2}\left(a x+\frac{4 b y}{\tau}\right)}\right. \\
& \left.\quad-i^{m+a} \frac{a \tau+4 b i}{m \tau}\left(m \tau e^{\frac{i \pi}{2}\left(a x-\frac{4 b y}{\tau}\right)}+(a \tau-4 b i) k e^{-\frac{i \pi}{2}\left(a x-\frac{4 b y}{\tau}\right)}\right)\right) e^{j \pi\left\langle\beta_{0}, z\right\rangle} \\
= & \frac{1}{2 \pi b m}\left(m \tau e^{\frac{i \pi}{2}\left(a x+\frac{4 b y}{\tau}\right)}+(a \tau+4 b i) k e^{\frac{-i \pi}{2}\left(a x+\frac{4 b y}{\tau}\right)}\right.
\end{aligned}
$$




$$
\begin{aligned}
& \left.-i^{m+a}\left((a \tau+4 b i) e^{\frac{i \pi}{2}\left(a x-\frac{4 b y}{\tau}\right)}+m \tau k e^{-\frac{i \pi}{2}\left(a x-\frac{4 b y}{\tau}\right)}\right)\right) e^{j \pi\left\langle\beta_{0}, z\right\rangle} \\
= & \frac{e^{\frac{i \pi a x}{2}}}{2 \pi b m}\left(e^{\frac{i 2 \pi b y}{\tau}}(m \tau+(a \tau+4 b i) k)\right. \\
& \left.-i^{m+a} e^{-\frac{i 2 \pi b y}{\tau}}(m \tau-(a \tau+4 b i) k) k\right) e^{\frac{j \pi m x}{2}} \\
= & \frac{1}{2 \pi b m}\left(e^{\frac{i 2 \pi b y}{\tau}} Y^{\prime}(x)+e^{-\frac{i 2 \pi b y}{\tau}} Y^{\prime \prime}(x)\right)
\end{aligned}
$$

with

$$
\begin{aligned}
Y^{\prime}(x)= & e^{\frac{i \pi a x}{2}}(m \tau+(a \tau+4 b i) k) e^{\frac{j \pi m x}{2}} \\
= & e^{\frac{i \pi a x}{2}}(m \tau+(a \tau+4 b i) k) \frac{e^{\frac{i \pi m x}{2}}(1-k)+e^{-\frac{i \pi m x}{2}}(1+k)}{2} \\
= & \frac{e^{\frac{i \pi}{2}(a+m) x}}{2}((m-a) \tau-4 b i)(1-k) \\
& \quad+\frac{e^{\frac{i \pi}{2}(a-m) x}}{2}((m+a) \tau+4 b i)(1+k) .
\end{aligned}
$$

Similarly

$$
\begin{aligned}
Y^{\prime \prime}(x)=i^{m+a} & \left(\frac{e^{\frac{i \pi}{2}(a+m) x}}{2}((m-a) \tau-4 b i)(1-k)\right. \\
& \left.-\frac{e^{\frac{i \pi}{2}(a-m) x}}{2}((m+a) \tau+4 b i)(1+k)\right) .
\end{aligned}
$$

Finally

$$
\begin{aligned}
Y_{\gamma}(z) & =\frac{1}{4 \pi b m}\left(\left(e^{\frac{i 2 \pi b y}{\tau}}+i^{m+a} e^{-\frac{i 2 \pi b y}{\tau}}\right) e^{\frac{i \pi}{2}(a+m) x}((m-a) \tau-4 b i)(1-k)\right. \\
& \left.+\left(e^{\frac{i 2 \pi b y}{\tau}}-i^{m+a} e^{-\frac{i 2 \pi b y}{\tau}}\right) e^{\frac{i \pi}{2}(a+m) x}((m+a) \tau+4 b i)(1+k)\right) .
\end{aligned}
$$

If $m+a \equiv 0 \bmod 4$,

$$
\begin{aligned}
Y_{\gamma}= & \frac{1}{2 \pi b m}\left(\cos \frac{2 \pi b y}{\tau} e^{\frac{i \pi}{2}(a+m) x}((m-a) \tau-4 b i)(1-k)\right. \\
& \left.+i \sin \frac{2 \pi b y}{\tau} e^{\frac{i \pi}{2}(a-m) x}((m+a) \tau+4 b i)(1+k)\right),
\end{aligned}
$$


while $m+a \equiv 2 \bmod 4$ gives a similar expression for $Y_{\gamma}$ where only $\cos$ and $i \sin$ are exchanged. We may sum up saying that $Y_{\gamma}$ has the form

$$
Z_{p, q, b}(x, y)=\cos \frac{2 \pi b y}{\tau} e^{2 i p \pi x} C_{1}+i \sin \frac{2 \pi b y}{\tau} e^{i q \pi x} C_{2},
$$

where

- if $m+a \equiv 0 \bmod 4,(p, q)=\left(\frac{a+m}{2}, \frac{a-m}{2}\right)$ and

$$
\left\{\begin{array}{l}
C_{1}=-\frac{q \tau+i 2 b}{\pi b m}(1-k) \\
C_{2}=\frac{p \tau+i 2 b}{\pi b m}(1+k)
\end{array}\right.
$$

- if $m+a \equiv 2 \bmod 4,(p, q)=\left(\frac{a-m}{2}, \frac{a+m}{2}\right)$ and

$$
\left\{\begin{array}{l}
C_{1}=\frac{q \tau+i 2 b}{\pi b m}(1+k) \\
C_{2}=-\frac{p \tau+i 2 b}{\pi b m}(1-k)
\end{array} .\right.
$$

Notice that in both cases $p \equiv 0 \bmod 2$ and $q \equiv 1 \bmod 2$. We conclude with the following property, obvious on the form above

$$
Z_{p, q, b}\left(x+\frac{k}{2 p}, \ell \tau / 2\right)=(-1)^{k+\ell b} Z_{p, q, b}(x, 0) .
$$

Step 3: Self-intersections.

Consider a Klein bottle, hence a $\mathbb{H}$-linear combination of terms $Z_{p_{i}, q_{i}, b_{i}}$. The condition (16) implies

$$
b_{i}^{2} p_{j} q_{j}=b_{j}^{2} p_{i} q_{i}
$$

Assume some optimal vertical periodicity; in particular the $b_{i}$ 's are not all even (otherwise we change the lattice $\Gamma$ ). Now

$$
Z_{p_{i}, q_{i}, b_{i}}\left(x+\frac{1}{2}, \ell_{i} \tau / 2\right)=(-1)^{p_{i}+\ell_{i} b_{i}} Z_{p_{i}, q_{i}, b_{i}}(x, 0) .
$$

We claim that there exist an integer $\ell$ such that

$$
p_{i}+\ell b_{i} \equiv 0 \bmod 2 .
$$

Assume - up to reindexing - that $b_{1} \equiv 1$ (all equivalences are taken mod 2) then $\ell \equiv p_{1}$ works. If for some $i, b_{i} \equiv 1$ then (17) implies $p_{i} \equiv p_{1}$ and equation (18) holds. If on the contrary $b_{i} \equiv 0$, then $p_{i} \equiv 0$ necessarily, and (18) holds again.

We conclude that $Z_{p_{i}, q_{i}, b_{i}}\left(x+\frac{1}{2}, \ell \tau / 2\right)=Z_{p_{i}, q_{i}, b_{i}}(x, 0)$ for any $i$; the same holds for any linear combination. So the Klein bottle is not embedded. Notice that one may construct Klein bottles having only that self-intersection, and no other, but not on a square lattice.

An example. Taking $\tau=1$ and $a=3, b=1, m=5$, we obtain:

$$
Y_{\gamma}=\frac{1-i 2}{5 \pi}\left(\cos 2 \pi y e^{i 4 \pi x}(1-k)-\sin 2 \pi y e^{-i \pi x}(1+k)\right) .
$$




\section{References}

[B1] A.I. Bobenko, Surfaces in terms of 2 by 2 matrices. Old and new integrable systems, in Harmonic Maps and Integrable Systems, Vieweg, Aspects of Mathematics E23 (1994), p. 83-127.

[B2] A.I. Bobenko, Exploring Surfaces through Methods from the Theory of Integrable Systems. Lectures on the Bonnet Problem, Lectures given at the School on Differential Geometry (April 1999), ICTP Trieste.

[CM] B.-Y. Chen, J.-M. Morvan, Deformations of isotropic submanifolds in Kähler manifolds, J. of Geometry and Physics 13 (1994), 79-104.

[CU] I. Castro, F. Urbano, Examples of unstable Hamiltonian-minimal Lagrangian tori in $\mathbb{C}^{2}$, Compositio Mathematica 111 (1998), 1-14.

[HL] R. Harvey, H. B. Lawson, Calibrated geometries, Acta Mathematica 148 (1982), 47-157.

[HR] F. Hélein, P. Romon, Hamiltonian stationary Lagrangian surfaces in $\mathbb{C}^{2}$, preprint CMLA 1999, submitted to Comm. Analysis Geo.

[Ke] K. Kenmotsu, Weierstrass formula for surfaces, Math. Ann. 245 (1986), 89-99.

[Ko] B. G. Konopelchenko, Induced surfaces and their integrable dynamics, Studies in Appl. Math. 96 No.1 (1996), 9-51.

[KoT1] B. G. Konopelchenko, I. Taimanov, Constant curvature surfaces via integrable dynamical system, J. Phys. A, Math. Gen. 29 No.6 (1996), 1261-2165.

[KoT2] B. G. Konopelchenko, I. Taimanov, Generalized Weierstrass formulae, soliton equations and Willmore surfaces I. Tori of revolution and the mKdV equation, preprint.

[KuS] R. Kusner, N. Schmitt, The spinor representation of minimal surfaces, GANG preprint (1996).

[O1] Y.G. Oh, Second variation and stabilities of minimal Lagrangian submanifolds in Kähler manifolds, Invent. Math. 101 (1990), 501-519.

[O2] Y.G. Oh, Volume minimization of Lagrangian submanifolds under Hamiltonian deformations, Math. Z. 212 (1993), 175-192.

[R] J. Richter, Conformal Maps of a Riemannian Surface into the Space of Quaternions, PhD thesis, Technische Universität Berlin, 1997.

[SW] R. Schoen, J. Wolfson, Minimizing volume among Lagrangian submanifolds, in Differential equations: La Pietra 1996 (Florence), 181-199, Proc. Sympos. Pure Math., 65, Amer. Math. Soc., Providence, RI (1999).

[T1] I. A. Taimanov, The Weierstrass representation of closed surfaces in $\mathbb{R}^{3}$, Funktsional. Anal. i Prilozhen. 32 no. 4 (1998), 49-62; translation in Funct. Anal. Appl. 32 no. 4 (1998), 258-267.

[T2] I. A. Taimanov, The Weierstrass representation of spheres in $\mathbb{R}^{3}$, the Willmore number and soliton sphere, preprint 302 SFB 288 (1997) and Tr. Mat. Inst. Steklova 225 (1999), Solitony Geom. Topol. na Perekrest., 339-361.

[V] C. Viterbo, Metric and isoperimetric problems in symplectic geometry, J. Amer. Math. Soc. 13 no. 2 (2000), 411-431 (electronic).

[W] J. Wolfson, Minimal Lagrangian diffeomorphisms and the Monge-Ampère equations, $J$. Differential Geometry 46 (1997), 335-373. 
Frédéric Hélein

Institut Universitaire de France

CMLA, ENS de Cachan

61 avenue du Président Wilson

F-94235 Cachan Cedex

France

e-mail: helein@cmla.ens-cachan.fr
Pascal Romon

CMLA, ENS de Cachan

61 avenue du Président Wilson

F-94235 Cachan Cedex

France

and

Université de Marne-la-Vallée

5 , boulevard Descartes

Champs sur Marne

F-77454 Marne-la-Vallée Cedex 2

France

e-mail: romon@cmla.ens-cachan.fr

(Received: January 25, 2000) 